\title{
About the Electrospray Ionization Source in Mass Spectrometry: Electrochemistry and On-chip Reactions
}

\author{
Michel Prudent ${ }^{\S}$, Manuel A. Méndez ${ }^{\mathrm{a}}$, Christophe Roussela, Bin Sư ${ }^{\mathrm{a}}$, Niels Lion ${ }^{\mathrm{a}}$, \\ Joël S. Rossier ${ }^{b}$, and Hubert H. Girault*a \\ $\S S C S$ Mettler-Toledo Award Winner (Oral Presentation)
}

\begin{abstract}
The present work shows that the electrochemical properties of electrospray ionization (ESI) can be used to add functions to the process. As example, we show how the choice of the electrode material can be used to study interactions between metal ions and biomolecules in mass spectrometry (MS). In positive ionization MS, an electrospray device acts as anode, which implies oxidation reactions. Sacrificial electrodes (made of copper or zinc) are used to supply the electrospray current and to produce cations that are able to react on-line with compounds of interest. Thus, the interactions between copper ions and ligands or peptides were investigated by using a copper electrode. Another example is the in situ electrogeneration of a dinuclear zinc(II) complex for the mass tagging of phosphopeptides when working with a zinc electrode. In order to perform these reactions on the same microchip, a dual-channel microsprayer was used, where one channel was dedicated to the tag electrogeneration and the other to the infusion of a phosphopeptides solution. Finally, this dual-channel microsprayer was used to study complexation at liquid-liquid interfaces in biphasic ESI-MS, such as thioether crowns and lead ions or peptides and phospholipids complexes. These examples illustrate the use of electrochemistry and on-chip reactions in ESI-MS analysis.
\end{abstract}

Keywords: Biomolecules · Electrochemistry · Electrospray ionization · Metal ions · Microchip

\section{Introduction}

Electrospray ionization (ESI) transfers charged molecules to the gas phase for mass analysis. Since its introduction in the 1980s the interest and popularity of ESI has increased rapidly. This technique has been explored since the 18th century with the pioneering work of the abbot Nollet, a French clerical who studied the effect of electricity on liquids. ${ }^{[1]}$ After different

\footnotetext{
${ }^{*}$ Correspondence: Prof. H. H. Girault ${ }^{\mathrm{a}}$

Tel.: + 41216933667

Fax: + 41216933145

E-mail: hubert.girault@epfl.ch

aLaboratoire d'Electrochimie Physique et Analytique

Ecole Polytechnique Fédérale de Lausanne

Station 6

$\mathrm{CH}-1015$ Lausanne

'DiagnoSwiss SA

route de l'lle au Bois,

$\mathrm{CH}-1870$ Monthey
}

discoveries and experiments, the electrospray was further developed and another step was made in the early 20th century by Zenely, who imaged the process. ${ }^{[2]}$ The observed conical shape came to be termed the Taylor cone based on the later theoretical work of Taylor in 1964. ${ }^{[3]}$ After all the physical investigations, electrospray as an ionization source for mass spectrometry (MS) was really introduced in 1968. Dole and coworkers used this technique to transfer high molecular weight polystyrene ions into the gas phase, ${ }^{[4]}$ and in the 1980s Fenn and coworkers presented a series of papers that permanently established electrospray as a tool to introduce dissolved analytes into the gas phase for mass analysis. ${ }^{[5]}$

Basically, this process is based on the application of high voltage to a solution. ${ }^{[6,7]}$ This creates a fine aerosol of highly charged droplets in a strong electric field. The liquid to be analyzed passes through a capillary and, at the tip, the high electric field induces the deformation of the polarized air-liquid interface into a conical shape. ${ }^{[3]}$ Then, the solution breaks up into a fine mist of charged droplets. The last step is the formation of gas-phase ions able to fly inside the MS in order to be analyzed.

Behind the ESI process, the nature of the source itself renders possible a lot of modifications for solution chemistry by taking advantage of its electrolytic nature or by driving in situ reactions and on-line coupling. ESI involves many redox reactions and can be considered as an electrochemical cell. ${ }^{[8]}$ Indeed, in order to compensate the departure of an ion from the Taylor cone and to sustain the electrospray current heterogeneous electron-transfer reactions must occur at the conductive contact to the solution. Electrochemistry is thus inherent to the operation of the source and a better use of the electrochemistry involved in ESI is of interest to promote and detect specific molecules. ${ }^{[8]}$

The electrochemical reactions inherent to the ESI source take place at the working electrode in contact with the solution. The potential of the emitter electrode is not fixed during ESI-MS experiments and this potential is adjusted to a certain level depending on the current density, the concentration and redox potential of the analytes, and so forth. ${ }^{[9]}$ In positive ionization mode, oxidation reactions occur in which the electrode material is also involved. Thus, the use of a sacrificial electrode will produce ions in solution, as shown by Blades et al. with zinc and stainless steel capillary tips. ${ }^{[10]}$ Van Berkel and coworkers have shown that the corrosion of the electrodes produces reactive ions, but, depending on the particular scenario analyzed and on the 
analyte chemical properties, it might also affect the appearance of the mass spectra acquired. ${ }^{[11]}$ In our lab, Rohner and Girault have coupled a sacrificial electrode with a microchip in order to study the complexation between two model peptides and different metal ions. ${ }^{[12]}$

Because of the flexibility of the ESI source several reactions can be achieved within the emitter by the use of diverse modifications. Microfluidic hyphenated with ESI-MS has been intensively developed in the last decade and has opened the door to a broad type of development.[13,14] For instance, polyimide or polyethyleneterphthalate (PET) microchips can be used as emitters for ESI-MS.[15-18] The desired microchannels can be drilled by using a UV excimer laser ablating a PET substrate ${ }^{[16]}$ or by plasma etching polyimide. ${ }^{[19]}$ Dayon and coworkers used this microsprayer to selectively mark cysteines in peptides with different hydroquinones by on-line electrochemicaltagging. [20,21]

The goal here is to add function to ESI (functional ESI - FESI) by taking advantage of the electrochemical aspects and of the source design in the particular case of bioinorganic studies (Fig. 1). First, sacrificial copper electrodes will be used in order to study copper-peptide complexes. Then, the mass tagging of phosphopeptides will be achieved by using a zinc electrode and also by taking benefit of the design of a dual-channel microsprayer, allowing in situ reactions. Finally, this microchip, containing two inlets, will allow us to build a biphasic electrospray ionization (BESI) source to study complexes involved at liquid-liquid interfaces

\section{Experimental}

Metal ion on-line complexation was carried out using a microspray interface described by Rohner and Girault.[12] It consists of a single microchannel polyimide microchip developed by DiagnoSwiss SA (Monthey, Switzerland).[15,19] A reservoir of polycarbonate was glued at the inlet of the microchannel. Samples in $50 / 50(v / v) \mathrm{MeOH} / \mathrm{H}_{2} \mathrm{O}$ were loaded in the reservoir in which an electrode

\begin{tabular}{|c|c|c|}
\hline & & Examples \\
\hline \multirow{3}{*}{ 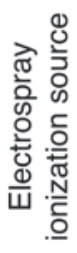 } & Electrochemistry & Copper complexes \\
\hline & $\mathrm{Red} \longrightarrow \mathrm{Ox}^{n+}+n e^{-}$ & Mass tagging of phosphopeptides \\
\hline & On-chip reactions & $\begin{array}{l}\text { Peptide- and metal-phospholipid } \\
\text { complexes }\end{array}$ \\
\hline
\end{tabular}

Fig. 1. Electrospray ionization source: interconnections between the inherent electrochemical properties of ESI and in situ reactions. The examples shown take advantage more or less of both properties. oxidation of uncomplexed copper(I) ions. On the other hand, when the experiments were performed in presence of a copper(II) salt and with a Pt electrode the 1:1 complex was much higher $(81 \%$ of relative abundance), but the $2: 1$ complex was still the most abundant. This formation of copper(I) complexes from a copper(II) salt can be explained by the reduction of $\mathrm{Cu}^{2+}$ to $\mathrm{Cu}^{+}$in gas phase. ${ }^{[28,29]} \mathrm{Cu}^{+}$ions formed in gas phase were able to react with ligands offering a better affinity. However, UV-vis experiments carried out after electrodissolution of copper in the spray solution containing BCP corroborated the formation of $\mathrm{Cu}^{+}$complexes in solution. ${ }^{[24]}$

Fig. 2 clearly highlights the behavior of the copper etching described above. In presence of a mixture of $\mathrm{BCP}$ and Hox, the copper complexes formed following the addition of a copper(II) salt or following the copper electrodissolution were studied. The experiment carried out with the copper electrode (Fig. 2) shows the formation of three complexes. The $2 \mathrm{BCP}-\mathrm{Cu}^{+}$complex is again the most abundant. Moreover, an interesting peak pattern appeared at $\mathrm{m} / \mathrm{z}=$ 568.3 Th (see bottom left on Fig. 2), which corresponds to the overlap of two isotopic distributions. The peak at $\mathrm{m} / z=567.1 \mathrm{Th}$ corresponds to a $\mathrm{Cu}^{2+}$ complex whereas the other at $\mathrm{m} / \mathrm{z}=568.3 \mathrm{Th}$ corresponds to a $\mathrm{Cu}^{+}$complex. The latter was not observed with the copper(II) salt (see bottom right on Fig. 2). It has to be also pointed out that the peak pattern is different when using a copper(II) salt; the $\mathrm{BCP}-\mathrm{CuO}^{+}$and $\mathrm{BCP}-\mathrm{Hox}-\mathrm{Cu}^{2+}$ complexes were the most abundant whilst the peak of $2 \mathrm{BCP}-\mathrm{Cu}^{+}$was weak. The electrochemical approach offers access to different complexes in solution, either $\mathrm{Cu}^{2+}$ or $\mathrm{Cu}^{+}$, without specific sample preparation.

The next step was the study of copperpeptide complexes electrogenerated from a sacrificial copper electrode. ${ }^{[23]}$ Amino acid residues have a specific affinity for cuprous or cupric ions and different complexes can be observed depending on the peptide sequences and the oxidation states of copper ions. In vivo, the anchor sites for $\mathrm{Cu}^{+}$include the thiol moieties of cysteines and those for $\mathrm{Cu}^{2+}$ include the imidazole residues of histidines. ${ }^{[30]}$ In the gas phase, the binding sites of $\mathrm{Cu}^{2+}$ are quite identical to those observed in solution whereas arginine becomes the preferred anchorage for $\mathrm{Cu}^{+}{ }^{[31]}$ Therefore, a histidine- and argininecontaining peptide, namely angiotensin III (angIII, RVYIHPF), was chosen to investigate the relative complexation properties of the two oxidation states. When using a copper electrode to study this peptide, it was found that $\mathrm{Cu}^{2+}$ anchored to the histidine and $\mathrm{Cu}^{+}$to the arginine. In comparison with this result, experiments run with a $\mathrm{Pt}$ electrode in presence of $\mathrm{CuCl}_{2}$ only re- 


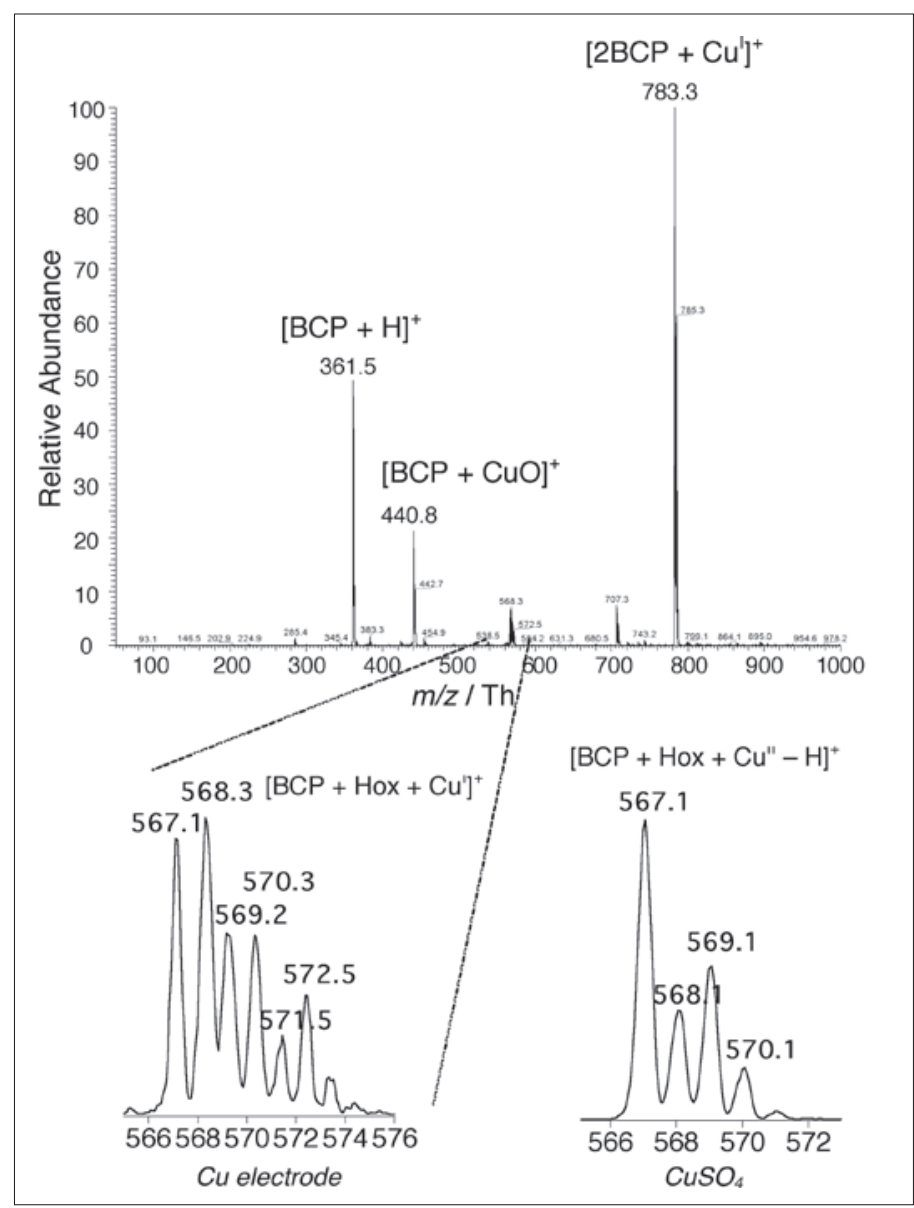

Fig. 2. Mass spectrum of a mixture of 8-hydroxyquinoline (Hox) and bathocuproine (BCP, $50 \mu \mathrm{M}$ each) in $50 / 50(\mathrm{v} / \mathrm{v}) \mathrm{MeOH} / \mathrm{H}_{2} \mathrm{O}$ obtained with a copper electrode after $7 \mathrm{~min}$ of experiments. The inset on the bottom right corresponds to the magnified part extracted from the mass spectrum of the same solution but electrosprayed with a Pt electrode in presence of $\mathrm{CuSO}_{4}$ at $50 \mu \mathrm{M}, t=6 \mathrm{~min}$. The inset on the bottom left is a zoom of the MS shown.

vealed the binding of $\mathrm{Cu}^{2+}$ to the histidine. Peptides fragmentation was also observed and related to $\mathrm{Cu}^{2+}$ ions. The same principle was applied to histidine-free peptide (e.g. bradykinin, RPPGFSPFR) and $\mathrm{Cu}^{2+}$ was found in the middle of the structure (i.e. GFSP) whereas $\mathrm{Cu}^{+}$anchored to both arginine residues. Afterward, Leuenkephalin (enke, YGGFL) was used as a histidine- and arginine-free sequence. The data observed with the copper electrode and the platinum electrode in presence of a copper(II) salt were similar, even though $\mathrm{Cu}^{+}$ions are preferentially formed due to the electrode oxidation.

Finally, the interactions of copper ions and cysteine-containing peptides were investigated. ${ }^{[22]}$ Copper is known to catalyze the oxidation of cysteine residues and $\mathrm{Cu}^{+}$ has a particular affinity for the thiol moiety. The complexation and the influences of copper ions electrogenerated from a copper electrode were tested on different cysteine-containing peptides. The main results were the addition of $\mathrm{Cu}^{+}$on residues such as cysteine and lysine, and peptide oxidation forming intra- or inter-molecular disulfide bridges. This oxidation was also observed in the absence of copper ions but in a weaker proportion. Moreover, the presence of copper ions enhanced the formation of inter-molecular disulfide bridges, which results in the enhancement of $2: 1$ (peptide to copper) complexes. Indeed, the complexation and the electron transfer reactions are the keys of the oxidation process, ${ }^{[32,33]}$ and the peptide structure plays an important role in disulfide bridges formation. As for the presence of $\mathrm{Cu}^{+}$ions, they were also observed when the peptides were mixed with a copper(II) salt. During cysteine oxidation, $\mathrm{Cu}^{2+}$ is reduced to $\mathrm{Cu}^{+}$, forming a cuprous biscysteine complex as the catalytic species. Electrogenerated copper ions can participate in the formation of this catalytic species, and $\mathrm{Cu}^{2+}$ ions also electrogenerated (even in presence of ligand specific for $\left.\mathrm{Cu}^{+}\right)^{[24]}$ can participate in the cysteine oxidation.

\section{Functional ESI: Tagging of Phosphopeptides}

Phosphorylation is a reversible modification that affects the folding and func- ion of proteins, and it is involved in many crucial cell functions. The identification of phosphorylation reactions is of importance for the determination of various mechanisms and is achieved, amongst other techniques, by MS. Dinuclear zinc(II) complex, namely 1,3-bis[bis(2-pyridylmethyl)amino]-propan-2-olato dizinc(II) (called thereafter tag), binds specifically to the phosphate moieties of phosphorylated compounds, inducing a mass shift, as shown by Takeda et al. in matrix-assisted laser desorption/ionization-MS. [34] This metallic tag made of zinc can be electrogenerated from a sacrificial zinc electrode and the ad hoc ligand. Thus, the idea is to carry out on the same microchip both the tag electrogeneration, by an in situ oxidation of a zinc electrode used to apply the electrospray current, and the phosphopeptides tagging. ${ }^{[26]}$ For this purpose, a dualchannel microsprayer made by DiagnoSwiss SA was used (Fig. 3). This device consists of a polyimide microchip with two microchannels etched on each side of the structure and connected only at the tip of the emitter. In this way, two liquids are mixed within the Taylor cone, formed during the ESI process.

As a model system, a mixture of two phosphorylated (p_angII, DRVpYIHPF and keratin K18, RPVSSAApSVYAGAC) and nonphosphorylated (angII, DRVY- 
IHPF and brady, RPPGFSPFR) peptides dissolved in pure water was put into a well stuck onto one inlet of the dual-channel microsprayer. The other well was filled with a solution of 2-propanol containing the ligand 1,3-bis[bis(2-pyridylmethyl)amino]2-propanol (LH, commercially available) where a zinc electrode was immersed. When the MS power supply was switched on, $\mathrm{Zn}^{2+}$ generation began, reacting with the ligand to form the tag in the well or in the downstream microchannel. The complex formed by the specific reaction of the phosphopeptides with the tag took place then within the Taylor cone and the products appeared in few minutes as a doubly charged species (Fig. 3). ${ }^{[26]}$ As a result, the relative abundance of the tagged p_angII and K18 increased with time. Adducts were present in a few minutes (even a few seconds for the tagged p_angII). Concentration of LH being constant, this increase is due to $\mathrm{Zn}^{2+}$ production and accumulation. Kebarle and coworkers have described the relationship between the $\mathrm{Zn}^{2+}$ concentration (following the oxidation of the electrode) and the spray current $i_{\mathrm{ES}} \cdot{ }^{[10]}$ The tag concentration calculated was in the micromolar range. ${ }^{[26]}$ The higher the current, the higher the $\mathrm{Zn}^{2+}$ concentration. Here, $i_{\mathrm{ES}}$ is defined (amongst other factors) by the distance between the chip and the entrance of the MS, but a compromise needs to be obtained between the current and the spectra quality. In spite of this relative low concentration, the phosphopeptides tagging occurs easily in a within few minutes. As a last point, the conversion from the untagged to the tagged phosphopeptides was higher than that expected for a total reaction and was related to an increase in ionization efficiency because of the addition of a triply charged tag, which is helpful for the identification of phosphopeptides.

The use of a zinc sacrificial electrode as an anode helps both the formation of the tag during the experiments thanks to zinc oxidation and the application of the spray current. The electrogenerated dinuclear zinc(II) complexes react with the phosphopeptides without any preparation of the complex before the analysis and avoid the contamination of counter ions used in the chemical tag synthesis. ${ }^{[26]}$ The drawback of such electrogeneration remains the relatively low tag production, which could be solved by lowering sample concentration or performing the electrogeneration in an electrochemical cell prior to the microchip inlet. ${ }^{[35,36]}$

\section{Biphasic ESI: Study of Interfacial Complexation}

Biphasic liquid systems have found many applications ranging from the clas-

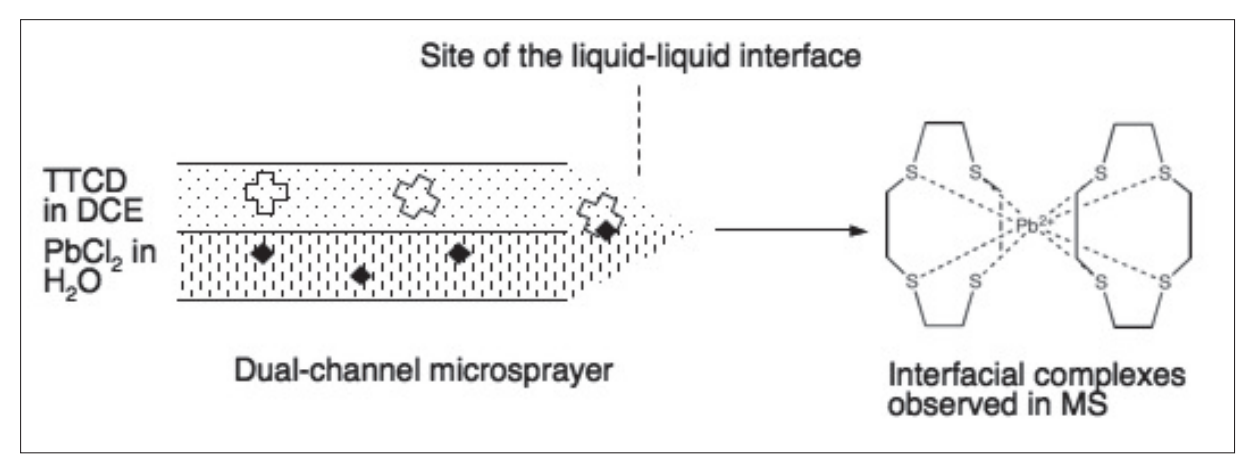

Fig. 4. Illustration of the BESI source. Example of complexes between thioether crown (TTCD) in DCE and lead chloride in water.

sical solvent extraction to the study of heterogeneous reactions via the mimicking of biological interfaces. Only a few studies reported the use of biphasic system in $\mathrm{MS}^{[37]}$ and the dual sprayer described above allows us to put into contact two immiscible liquids to study interfacial complexation in MS (Fig. 4). ${ }^{[25]}$ This microchip was fixed in a holder connected to a syringe pump that was able to hold two syringes to introduce two different solutions, e.g. water and 1,2-dichloroethane (DCE). The high voltage was applied to the stainless steel needle of the syringe containing the aqueous solution. A stable electrospray of two immiscible liquids was thus obtained over a sustained period, the limitation being the volume of solution. Hence, liquid-liquid interfaces are formed, enabling the study of chemical reactions under these conditions.

As a proof-of-concept, reactions between thioether crowns $(1,4,7,10$-tetrathiacyclododecane, TTCD, and 1,4,7,10,13,16-hexathiacyclooctadecane, HTCO) dissolved in DCE and lead chloride in water were investigated. ${ }^{[25]} \mathrm{Com}-$ plexes between the ligand and the ion were observed such as [HTCO-Pb $\left.{ }^{\mathrm{II}} \mathrm{Cl}\right]^{+}$. The formation of 1:1 (ligand to metal) and 2:1 complexes were also achieved with TTCD. As another example the reaction between an ether crown, dibenzo-18-crown-6 (DB18C6), in DCE and a dipeptide (FF) in acidified water $(1 \% \mathrm{AcOH})$ was successfully carried out with this BESI source. Different kinds of complexes were thus obtained, showing the feasibility of this new type of experiment. According to these results, two hypotheses about the reactions mechanisms can be written down, i.e. the adsorption and/or extraction of ion pairs, and the complex formation and adsorption at the interface. ${ }^{[25]}$ In addition, the intense mixing within the Taylor cone region should produce several interfaces, and droplets containing the two phases might be formed, the most probable one being an aqueous droplet covered by an organic solvent shell.

Finally, a phospholipid (L- $\alpha$-dipalmitoyl phosphatidylcholine, DPPC), known to adsorb at liquid-liquid interfaces, and its interactions with peptides and metal ions have been successfully studied with the BESI source. ${ }^{[38]}$ DPPC molecules were dissolved in DCE and put in contact with acidified water $(1 \%$ of $\mathrm{AcOH})$ within the Taylor cone. The results showed the protonated monomer and dimer. The mass spectra thus obtained offer a better quality and higher peak abundances compared to standard lipid analysis. The interactions between phospholipids and amino acids or a dipeptide have been tested with two polar amino acids, one basic (Lys) and one acidic (Glu) and a hydrophobic dipeptide (FF). In general, from the obtained mass spectra, it was always possible to observe the peaks of both reactants (except for Glu), but the complexes between DPPC and the amino acids or the dipeptide were hardly observed, unless the heated capillary temperature be decreased to $100{ }^{\circ} \mathrm{C}$ instead of $200{ }^{\circ} \mathrm{C}$. Not only basic amino acids are capable of forming complexes with DPPC, but also hydrophobic species as shown for FF. This fact suggests that hydrophobic interactions also play an important role in the complexation mechanism. To verify this point, the complexation of DPPC with two peptides were compared, one of which is composed of mainly hydrophobic amino acids (enke) and the other contains basic amino acids in its sequence (angIII). DPPC-angIII complexes were present in the mass spectrum as a singly- and doubly-charged noncovalent complex, and as $[2 \mathrm{DPPC} \text {-angIII }+2 \mathrm{H}]^{2+}$. As for enke, it formed a noncovalent complex with one DPPC. Whereas the abundance of amino acid-phospholipid complexes decreased when increasing the temperature, the relative abundance of peptide-phospholipid complexes increased and then starting to decrease beyond $160{ }^{\circ} \mathrm{C} .{ }^{[38]}$ This stability over the whole temperature range of the heated capillary tested exhibited a typical parabolic shape and can be explained by the balance between the quantity of gasphase ions produced and the transmission to the analyzer. An increase in the length of the peptide produced more stable com- 
plexes with DPPC due to the extended hydrophobic interactions.

We are currently investigating the metal-phospholipid complexes in our lab. As far as we know, clusters of DPPCs bind to one metal ion and are observed with $\mathrm{Na}^{+}$, $\mathrm{K}^{+}, \mathrm{Ca}^{2+}, \mathrm{Cu}^{+/ 2+}$ and $\mathrm{La}^{3+}$. In addition, the number of DPPC increases with the valence of the ions.

Beyond the advantage of filling different liquids in on-line ESI-MS, the BESI source developed here points out the reactivity of compounds from two different phases in ESI-MS. Moreover, the correlation between the MS data and electrochemical experiments at the liquid-liquid interface can be achieved. This is currently under development in our laboratory. ${ }^{[38]}$

\section{Conclusions}

Electrochemistry and on-chip reactions were central to these studies with the objective of analyzing bioinorganic compounds. Sacrificial electrodes give the advantage of in situ generation of metal ions which are able to react with other compounds. The latter implies the absence of prior salt addition or counter ions influence. Fundamental studies and development of this approach as an analytical tool for specific purposes are two challenging ways that could follow such research. The chip design allows different kind of reactions to be performed successfully and could be used in further experiments either for on-line derivatization or for studying interfacial reactions.

The implications of metal ions in biological systems are investigated with different methods and their studies are of interest both for understanding and revealing biological issues and for the development of metal-based drugs. The exploration of the electrospray ionization mass spectrometry was used with the goal of demonstrating the feasibility of such new approaches in bioinorganic analyses.

\section{Acknowledgments}

The Fonds National Suisse pour la Recherche Scientifique is thanked for financial support through the grant no 200020-113413/1. The authors also thank Fernando Cortés Salazar for discussions.

Received: January 14, 2009

[1] J. A. Nollet, 'Recherches sur les causes particulières des phénomènes électriques', 1ère ed., Chez les frères Guerin, Paris, 1749.

[2] J. Zeleny, Phys. Rev. 1917, 10, 1.

[3] G. Taylor, Proc. R. Soc. London, Ser. A 1964, $280,383$.

[4] M. Dole, L. L. Mack, R. L. Hines, J. Chem. Phys. 1968, 49, 2240.

[5] J. B. Fenn, M. Mann, C. K. Meng, S. F. Wong, C. M. Whitehouse, Science 1989, 246, 64.

[6] T. C. Rohner, N. Lion, H. H. Girault, Phys. Chem. Chem. Phys. 2004, 6, 3056.

[7] R. B. Cole, 'Electrospray Ionization Mass Spectrometry: Fundamentals Instrumentation \& Applications', 1st ed., John Wiley \& Sons, New York, NY, 1997.

[8] G. J. Van Berkel, V. Kertesz, Anal. Chem. 2007, 79, 5510 .

[9] G. J. Van Berkel, F. M. Zhou, Anal. Chem. 1995, 67, 2916.

[10] A. T. Blades, M. G. Ikonomou, P. Kebarle, Anal. Chem. 1991, 63, 2109.

[11] G. J. Van Berkel, K. G. Asano, P. D. Schnier, J. Am. Soc. Mass Spectrom. 2001, 12, 853.

[12] T. C. Rohner, H. H. Girault, Rapid Commun. Mass Spectrom. 2005, 19, 1183.

[13] N. Lion, T. C. Rohner, L. Dayon, I. L. Arnaud, E. Damoc, N. Youhnovski, Z. Y. Wu, C. Roussel, J. Josserand, H. Jensen, J. S. Rossier, M. Przybylski, H. H. Girault, Electrophoresis 2003, 24, 3533

[14] S. Koster, E. Verpoorte, Lab Chip 2007, 7, 1394.

[15] V. Gobry, J. Van Oostrum, M. Martinelli, T. C. Rohner, F. Reymond, J. S. Rossier, H. H. Girault, Proteomics 2002, 2, 405.

[16] T. C. Rohner, J. S. Rossier, H. H. Girault, Anal. Chem. 2001, 73, 5353.
[17] M. Abonnenc, L. Dayon, B. Perruche, N. Lion, H. H. Girault, Anal. Chem. 2008, 80, 3372.

[18] L. Dayon, M. Abonnenc, M. Prudent, N. Lion, H. H. Girault, J. Mass Spectrom. 2006, 41, 1484.

[19] J. S. Rossier, C. Vollet, A. Carnal, G. Lagger, V. Gobry, H. H. Girault, P. Michel, F. Reymond, Lab Chip 2002, 2, 145

[20] L. Dayon, C. Roussel, M. Prudent, N. Lion, H. H. Girault, Electrophoresis 2005, 26, 238.

[21] C. Roussel, L. Dayon, N. Lion, T. C. Rohner, J. Josserand, J. S. Rossier, H. Jensen, H. H. Girault, J. Am. Soc. Mass Spectrom. 2004, 15 1767.

[22] M. Prudent, H. H. Girault, Metallomics 2009, 1, 157.

[23] M. Prudent, H. H. Girault, J. Am. Soc. Mass Spectrom. 2008, 19, 560.

[24] M. Prudent, C. Roussel, H. H. Girault, Electrochem. Commun. 2007, 9, 2067.

[25] M. Prudent, M. A. Méndez, H. H. Girault, Anal. Sci. 2008, 24, 1399.

[26] M. Prudent, J. S. Rossier, N. Lion, H. H. Girault, Anal. Chem. 2008, 80, 2531.

[27] E. Mattsson, J. O. Bockris, Trans. Faraday Soc. 1959, 55, 1586.

[28] D. Schröder, M. C. Holthausen, H. Schwarz, J. Phys. Chem. B 2004, 108, 14407.

[29] H. Lavanant, H. Virelizier, Y. Hoppilliard, J. Am. Soc. Mass Spectrom. 1998, 9, 1217.

[30] R. M. Roat-Malone, 'Bioinorganic Chemistry', 1st ed., John Wiley \& Sons, Hoboken, 2002.

[31] B. A. Cerda, C. Wesdemiotis, J. Am. Chem. Soc. 1995, 117, 9734

[32] L. Pecci, G. Montefoschi, G. Musci, D. Cavallini, Amino Acids 1997, 13, 355.

[33] A. V. Kachur, C. J. Koch, J. E. Biaglow, Free Radic. Res. 1999, 31, 23.

[34] H. Takeda, A. Kawasaki, M. Takahashi, A. Yamada, T. Koike, Rapid Commun. Mass Spectrom. 2003, 17, 2075.

[35] X. M. Xu, W. Z. Lu, R. B. Cole, Anal. Chem. 1996, 68, 4244.

[36] G. J. Van Berkel, K. G. Asano, M. C. Granger, Anal. Chem. 2004, 76, 1493.

[37] H. Watarai, A. Matsumoto, T. Fukumoto, Anal. Sci. 2002, 18, 367.

[38] M. A. Méndez, M. Prudent, B. Su, H. H. Girault, Anal. Chem. 2008, 80, 9499. 